\title{
THE HISTORY OF AN HOUR
}

VAIN is the wish to try rhyming it, writing it! Pen cannot weld into words what it was;

Time will be squandered in toil at inditing it;

Clear is the cause!

Yea, 'twas too satiate with soul, too ethereal;

June-morning scents of a rose-bush in flower

Catch in a clap-net of hempen material;

So catch that hour!

Human Shows, Far Phantasies (1925) 